\title{
Loquat (Eriobotrya japonica) extract prevents dexamethasone-induced muscle atrophy by inhibiting the muscle degradation pathway in Sprague Dawley rats
}

\author{
KYUNG KYUN NOH ${ }^{1}$, KI WUNG CHUNG ${ }^{1}$, BOKYUNG SUNG ${ }^{1}$, MIN JO KIM ${ }^{1}$, CHAN HUM PARK ${ }^{1}$, \\ CHANGSHIN YOON $^{1}$, JAE SUE CHOI ${ }^{2}$, MI KYUNG KIM ${ }^{3}$, CHEOL MIN KIM ${ }^{4,5}$, \\ NAM DEUK KIM ${ }^{1}$ and HAE YOUNG CHUNG ${ }^{1}$
}

\footnotetext{
${ }^{1}$ Department of Pharmacy, Molecular Inflammation Research Center for Aging Intervention, Pusan National University, Busan 609-735; ${ }^{2}$ Department of Food Science and Nutrition, Pukyong National University, Busan 608-737;

${ }^{3}$ Longevity Life Science and Technology Institute, Pusan National University, Busan 609-735;

${ }^{4}$ Department of Biochemistry, Pusan National University School of Medicine, Yangsan, Gyeongsangnam-do 626-770;

${ }^{5}$ Research Center for Anti-Aging Technology Development, Busan 609-735, Republic of Korea
}

Received November 29, 2014; Accepted May 8, 2015

DOI: $10.3892 / \mathrm{mmr} .2015 .3821$

\begin{abstract}
In the Orient, loquat (Eriobotrya japonica) extract (LE) is widely used in teas, food and folk medicines. The leaves of the loquat tree have been used for generations to treat chronic bronchitis, coughs, phlegm production, high fever and gastroenteric disorders. One of the major active components of loquat leaves is ursolic acid, which was recently investigated in the context of preventing muscle atrophy. The present study investigated the therapeutic potential of LE on dexamethasone-induced muscle atrophy in rats. Daily intraperitoneal injections of dexamethasone caused muscle atrophy and evidence of muscle atrophy prevention by LE was demonstrated using various assays. In particular, dexamethasone-induced grip strength loss was alleviated by LE and the increase in serum creatine kinase activity, a surrogate marker of muscle damage, caused by dexamethasone injection was reduced by LE. Western blot analysis and immunoprecipitation demonstrated that dexamethasone markedly increased the protein expression levels of muscle ring finger 1 (MuRF1), which causes the ubiquitination and degradation of myosin heavy chain (MyHC), and decreased the protein expression levels of MyHC as well as increased the ubiquitinated MyHC to MyHC ratio. However, LE reduced the dexamethasone-induced protein expression levels of MuRF1 and ubiquitinated MyHC. Additional experiments revealed
\end{abstract}

Correspondence to: Professor Hae Young Chung, Department of Pharmacy, Molecular Inflammation Research Center for Aging Intervention, Pusan National University, 63 Beon-gil 2, Busan 609-735, Republic of Korea

E-mail: hyjung@pusan.ac.kr

Key words: forkhead box O1, loquat extract, ursolic acid, muscle ring finger 1, muscle atrophy, muscle degradation that LE supplementation inhibited the nuclear translocation of FoxO1 induced by dexamethasone. These findings suggested that LE prevented dexamethasone-induced muscle atrophy by regulating the FoxO1 transcription factor and subsequently the expression of MuRF1.

\section{Introduction}

Loquat (Eriobotrya japonica) is a tree which belongs to the Rosaceae family and is native to central China (Chongqing and Hubei provinces). In folk medicine, loquat leaves are used to treat various skin diseases, itching, coughs and nausea $(1,2)$. Furthermore, the terpenoids isolated from loquat leaves have been reported to possess anti-tumor, anti-viral and anti-inflammatory properties $(3,4)$. In particular, ursolic acid has been reported to be a major active component of loquat leaf extract (LE), with one gram of loquat leaves containing $\sim 1.5 \mathrm{mg}$ ursolic acid and $0.7 \mathrm{mg}$ oleanolic acid $(4,5)$. In a mechanistic study, it was revealed that ursolic acid inhibits cell cycle progression in the G1 phase and triggers apoptosis $(6,7)$. It has been previously demonstrated that ursolic acid inhibits muscle atrophy by regulating insulin/insulin-like growth factor (IGF)-1 signaling (8).

Skeletal muscle atrophy can result from several conditions, including sepsis, cancer, renal failure, glucocorticoid excess, denervation, muscle disuse and the aging process. This muscle atrophy results from an imbalance between the rates of protein synthesis and breakdown. Previous studies have reported that muscle ring finger 1 (MuRF1) and muscle atrophy F box (MAFbx) are upregulated in catabolic states $(9,10)$. Furthermore, MuRF1 and MAFbx have been demonstrated to be upregulated in models of atrophy addressing muscle disuse, hind limb suspension, denervation, glucocorticoid or interleukin-1 treatment, fasting, cancer cachexia, diabetes and renal failure. In addition, MuRF1 and MAFbx were revealed to target myofibrillar proteins and hypertrophic genes (11-13). These findings suggested that these two muscle-specific 
E3 ligases have distinct roles in muscle wasting. In addition, as muscle wasting results from increased proteolysis and reduced protein synthesis in most catabolic states, they appear to contribute to muscle mass depletion in a coordinated manner.

Previous studies suggested that members of the forkhead box $\mathrm{O}$ (FoxO) transcription factor family are important in muscle atrophy. In fact, FoxO factors have been reported to stimulate the expression of MuRF1 and increase protein degradation, and the activation of FoxO was demonstrated to cause significant myotube and mature muscle fiber atrophy, whereas the inhibition of FoxO reduced the induction of MuRF1 (14,15). In another previous study, transgenic mice overexpressing FoxO1 in skeletal muscle were revealed to weigh less compared with the wild-type controls (16).

Ursolic acid was recently identified as a modulator of phosphoinositide 3-kinase/Akt/FoxO signaling, and therefore, an inhibitor of muscle atrophy and the expression of MuRF1 (8). The aim of the present study was to investigate the effects of loquat extract, which contains ursolic acid, on the degradation of myosin heavy chain (MyHC) via MuRF1/FoxO1 signaling in the dexamethasone-induced muscle atrophy model, in order to evaluate the suitability of loquat extract to be used as a chemopreventive agent against muscle aging and/or as a therapeutic agent against skeletal muscle atrophy in the elderly.

\section{Materials and methods}

Chemicals. Dried loquat leaves were purchased from Pure Mind Co. (Daegu, Korea) and LE was prepared as previously described with slight modifications (17). Unless otherwise stated, all chemical reagents were obtained from Sigma-Aldrich (St Louis, MO, USA). Primary antibodies against MyHC (cat. no. sc-20641), MuRF1 (cat. no. sc-32920), MAFbx (cat. no. sc-33782), FoxO1 (cat. no. sc-67140), $\alpha$-tubulin (cat. no. sc-5286), transcription factor (TF)-IIB (cat. no. sc-225) and ubiquitin (cat. no. sc-271289), and secondary goat anti-rabbit immunoglobulin (Ig)G (cat. no. sc-2004) and anti-mouse IgG conjugated to horseradish peroxidase (cat. no. sc-2031) were obtained from Santa Cruz Biotechnology, Inc. (Dallas, TX, USA). Polyvinylidene difluoride (PVDF) membranes were obtained from EMD Millipore (Bedford, MA, USA).

Animal experiments. All animal studies were approved by the Institutional Animal Care Committee of Pusan National University (Busan, Korea) and were performed in accordance with the guidelines for animal experimentation issued by Pusan National University (Busan, Korea). Sprague Dawley rats (six weeks old) were obtained from Samtako (Osan, Korea). The rats were maintained in controlled environmental conditions $\left(23 \pm 1^{\circ} \mathrm{C}, 55 \pm 5 \%\right.$ relative humidity) under a $12-\mathrm{h}$ light/dark cycle with ad libitum access to water and a standard laboratory diet. The animals were randomly divided into five groups of six animals following a two-week acclimation period. Dexamethasone $(600 \mu \mathrm{g} / \mathrm{kg}$ body mass) or normal saline were intraperitoneally injected once daily for five consecutive days. The other compounds were administered by oral gavage. The animals were divided into groups based on the treatment type, as follows: Leucine $(600 \mathrm{mg} / \mathrm{kg} / \mathrm{day})$, ursolic acid (100 mg/kg/day) or LE (50 or $200 \mathrm{mg} / \mathrm{kg} / \mathrm{day}$ ). The leucine and ursolic acid supplementation groups were used as positive controls. At the end of the experimental period (day 5), forelimb grip strength was determined using a Grip Strength Meter equipped with a T-shaped pull bar (Model 1027SR; Columbus Instruments, Columbus, OH, USA). The rats were then sacrificed by decapitation and soleus and gastrocnemius muscles were rapidly removed. Muscle weight was measured immediately and the muscles were divided for biological analysis and histological examination.

Assay for creatine kinase activity. The serum creatine kinase (CK) activities were determined using a creatine kinase enzymatic assay kit (Bioo Scientific Corp, Austin, TX, USA) according to manufacturer's instructions. Serum was collected at sacrifice and stored at $-80^{\circ} \mathrm{C}$ until analysis. Briefly, $250 \mu \mathrm{l} \mathrm{CK}$ reagent was added to $5 \mu 1$ serum sample in a 96-well microplate. The CK activity was immediately measured twice with 5-min time intervals at $340 \mathrm{~nm}$ using a GENios microplate reader (Tecan, Salzburg, Austria). The activity of CK was calculated by subtracting the initial reading from the second reading and multiplying the absorbance increase by 2,186 (conversion factor) to the obtain $\mathrm{CK}$ activity (IU/1).

Histological analysis. The medial portions of soleus muscles were fixed in $10 \%$ formalin solution for $24 \mathrm{~h}$, routinely embedded in paraffin blocks, transversely sectioned $(3 \mu \mathrm{m})$ and stained with hematoxylin and eosin (H\&E). The muscle histologies were analyzed using an AE-31 light microscope (Motic, Hong Kong, China).

Tissue homogenization. Whole soleus muscle was homogenized in homogenizing buffer containing $50 \mathrm{mM}$ 4-(2-hydroxyethyl)-1-piperazineethanesulfonic acid (pH 7.4), $10 \mathrm{mM} \mathrm{KCl,} 2$ mM $\mathrm{MgCl}_{2}, 1 \mathrm{mM}$ dithiothreitol, 0.1 mMEDTA,0.1 mMphenylmethanesulfonylfluoride(PMSF), $20 \mathrm{mM}$ b-glycerophosphate, $20 \mathrm{mM} \mathrm{NaF}, 2 \mathrm{mM} \mathrm{Na} \mathrm{VO}_{4}$, $1 \mu \mathrm{M}$ pepstatin, $2 \mu \mathrm{M}$ leupeptin and $5 \mu \mathrm{M}$ aprotinin. The homogenates obtained were placed on ice for $15 \mathrm{~min}$. Thereafter, NP-40 was added at a final concentration of $1 \%$ (v/v) and the lysates were mixed for $15 \mathrm{sec}$. The nuclei were pelleted by centrifugation at $14,000 \mathrm{x}$ for $2 \mathrm{~min}$ at $4^{\circ} \mathrm{C}$. The supernatants were regarded as cytosolic fractions. The nuclear pellets were washed once, suspended in buffer containing $50 \mathrm{mM} \mathrm{KCl}, 300 \mathrm{mM} \mathrm{NaCl}, 0.1 \mathrm{mM}$ PMSF, $10 \%$ (v/v) glycerol, $20 \mathrm{mM} \beta$-glycerophosphate, $20 \mathrm{mM} \mathrm{NaF}, 2 \mathrm{mM} \mathrm{Na} \mathrm{VO}_{4}$, $1 \mu \mathrm{M}$ pepstatin, $2 \mu \mathrm{M}$ leupeptin and $5 \mu \mathrm{M}$ aprotinin, and maintained on ice for $30 \mathrm{~min}$. The suspensions were subsequently centrifuged at $14,000 \mathrm{x}$ g for $10 \mathrm{~min}$ at $4^{\circ} \mathrm{C}$ and the harvested supernatants were regarded as nuclear fractions. The protein concentrations were measured using the bicinchoninic acid method (Pierce ${ }^{\mathrm{TM}}$ BCA Protein Assay kit; Life Technologies, Carlsbad, CA, USA) using bovine serum albumin as a standard.

Immunoblotting. Nuclear or cytosolic proteins $(20-100 \mu \mathrm{g})$ were boiled for $5 \mathrm{~min}$ in gel-loading buffer containing $0.125 \mathrm{M}$ Tris- $\mathrm{HCl}$ (pH 6.8), 4\% SDS, 10\% 2-mercaptoethanol, $20 \%$ glycerol and $0.2 \%$ bromophenol blue, at a volume ratio of $1: 1$. The samples containing identical quantities of protein 
A

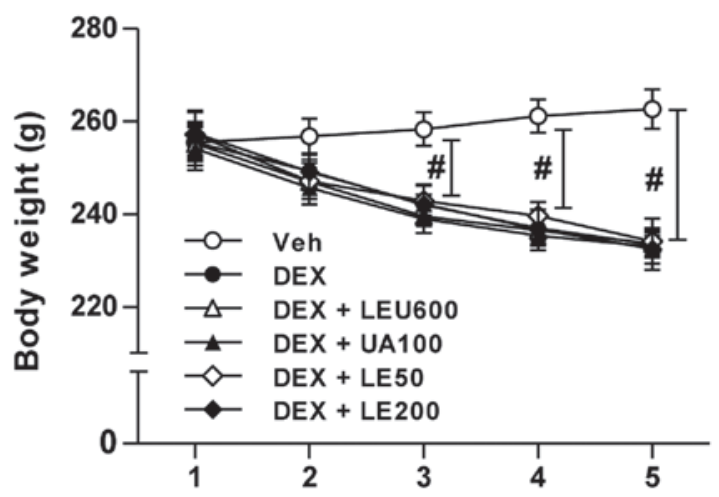

B

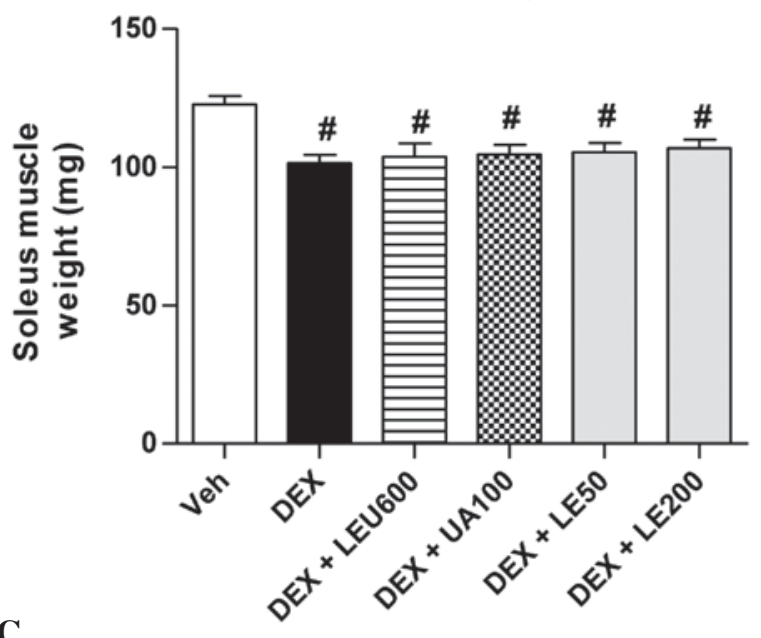

C

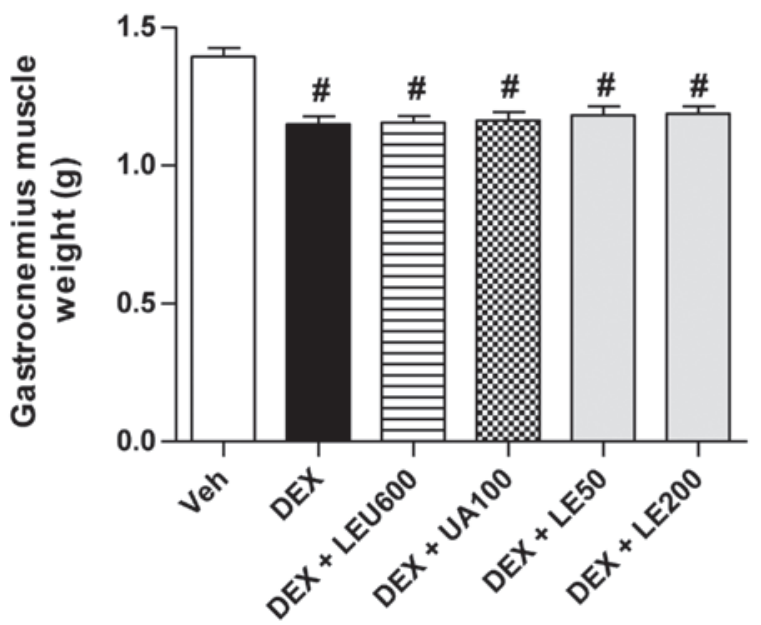

Figure 1. Changes in body, soleus muscle and gastrocnemius muscle weights in all groups during the five-day experimental period. (A) Body weight changes in each group. (B) Soleus muscle weight in the experimental groups. (C) Gastrocnemius muscle weight in each group. Values are expressed as the mean \pm standard error of the mean. Treatment doses are stated in $\mathrm{mg} / \mathrm{kg}$ body weight. $\mathrm{P}<0.05$, compared with the vehicle-treated group. DEX, dexamethasone; LEU, L-leucine; UA, ursolic acid; LE, loquat extract; Veh, vehicle-treated.

were subsequently separated using $8 \%-15 \%$ SDS-PAGE and transferred using a Bio-Rad Western system (Bio-Rad Laboratories, Hercules, CA, USA) onto PVDF membranes. The membranes were immediately placed in blocking buffer containing $5 \%$ non-fat milk in $10 \mathrm{mM}$ Tris ( $\mathrm{pH} 7.5)$, $100 \mathrm{mM} \mathrm{NaCl}$ and $0.1 \%$ Tween-20 for $1 \mathrm{~h}$. The membranes

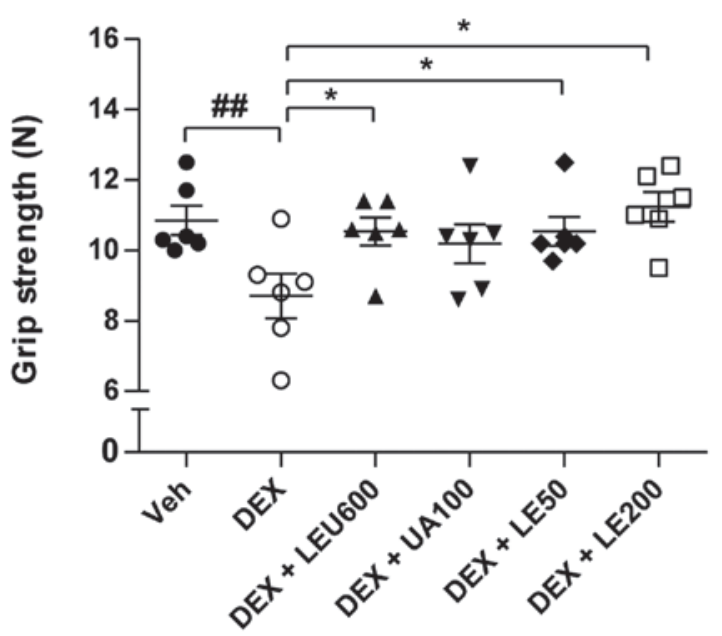

B

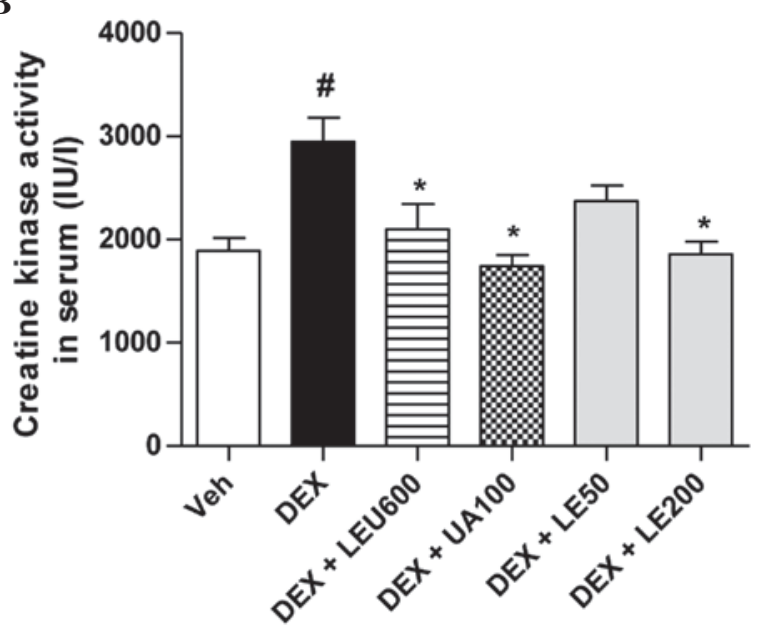

Figure 2. Forelimb grip strengths and serum creatine kinase activity. (A) Forelimb grip strength in the study groups prior to sacrification. Lines and bars represent the mean \pm standard error of the mean $(n=6)$. (B) Serum creatine kinase activity in the study groups. Serum creatine kinase activity was measured following sacrification. Values are expressed as the mean \pm standard error of the mean. Treatment doses are stated in $\mathrm{mg} / \mathrm{kg}$ body weight. ${ }^{\#} \mathrm{P}<0.05 ;{ }^{\#} \mathrm{P}<0.01$, compared with the DEX group and the vehicle-treated group; ${ }^{*} \mathrm{P}<0.05$, compared with the DEX group and the other four groups. DEX, dexamethasone; LEU, L-leucine; UA, ursolic acid; LE, loquat extract; Veh, vehicle-treated.

were incubated with the following primary antibodies at $4^{\circ} \mathrm{C}$ for $24 \mathrm{~h}$ : Anti-MyHC $(1: 1,000)$, anti-MuRF1 $(1: 1,000)$, anti-MAFbx $(1: 1,000)$, anti-FoxO1 (1:1,000), anti-TF-IIB $(1: 1,000)$, anti-ubiquitin $(1: 1,000)$, and anti- $\alpha$-tubulin $(1: 1,000)$. The membranes were then washed with TBS-Tween buffer (10 mM Tris, pH 7.5, $100 \mathrm{mM} \mathrm{NaCl}$ and $0.1 \%$ Tween-20) and incubated with horseradish peroxidase-conjugated secondary antibodies $(1: 5,000)$ for $1 \mathrm{~h}$ at room temperature. The resulting immunoblots were visualized using Western Bright Peroxide solution (Advansta, Menlo Park, CA, USA) on a Davinch-chemi ${ }^{\mathrm{TM}}$ Chemiluminescence Imaging system CAS-400 (Davinch-K, Seoul, Korea).

Immunoprecipitation. Homogenized tissue proteins were subjected to immunoprecipitation in a buffer, containing $40 \mathrm{mM}$ Tris- $\mathrm{HCl}$ ( $\mathrm{pH}$ 7.6), $120 \mathrm{mM} \mathrm{NaCl}$, $20 \mathrm{mM} \beta$-glycerophosphate, $20 \mathrm{mM} \mathrm{NaF}, 2 \mathrm{mM} \mathrm{Na}_{3} \mathrm{VO}_{4}$, 5 mM EDTA, 1 mM PMSF, $0.1 \%$ NP-40 containing leupeptin 

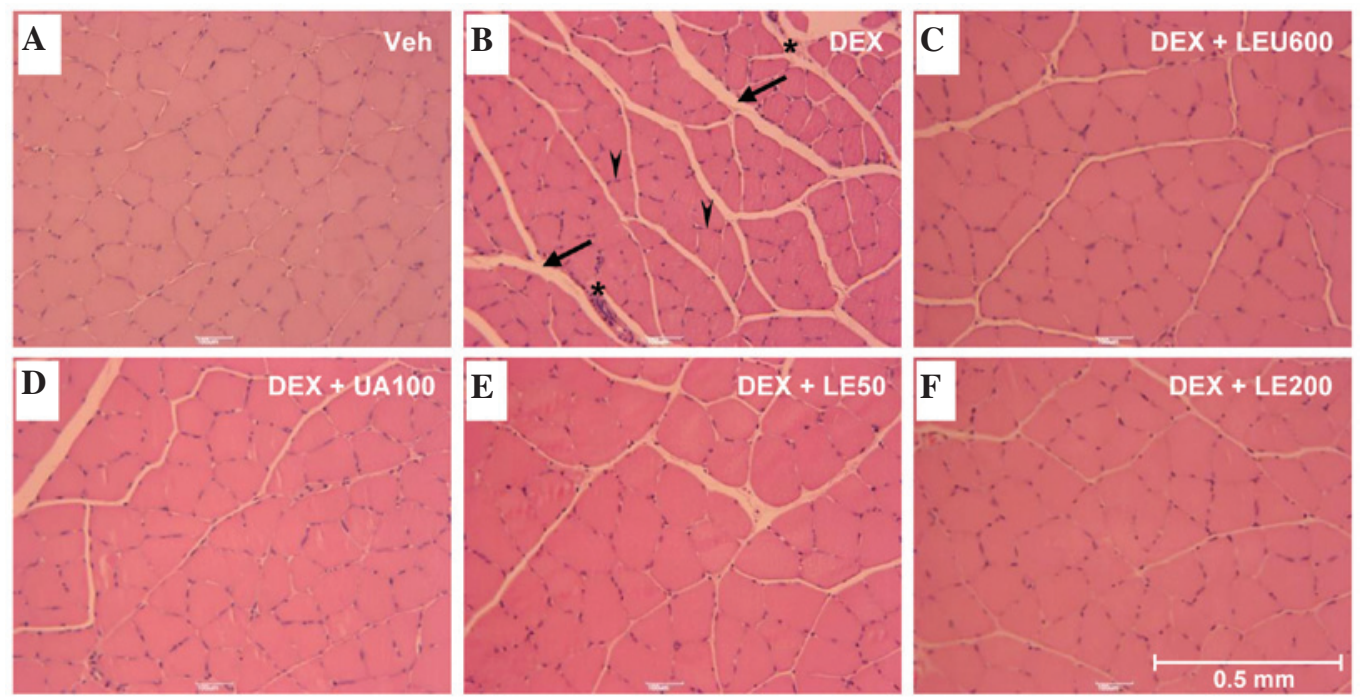

Figure 3. Histological analysis of soleus muscle cross sections. Hematoxylin and eosin-stained sections of excised soleus muscle tissues in the (A) Veh group (B) the DEX-treated group, (C) the DEX and LEU group, (D) the DEX and UA group and (E and F) the DEX and LE group. Arrows indicate the perimysium, arrow heads indicate the endomysium and asterisks indicate the angled and/or the degenerating fibers. Scale bar, $0.5 \mathrm{~mm}$. Treatment doses are stated in $\mathrm{mg} / \mathrm{kg}$ body weight. DEX, dexamethasone; LEU, L-leucine; UA, ursolic acid; LE, loquat extract; Veh, vehicle-treated.

$(2 \mu \mathrm{g} / \mathrm{ml})$, aprotinin $(1 \mu \mathrm{g} / \mathrm{ml})$ and pepstatin $\mathrm{A}(1 \mu \mathrm{g} / \mathrm{ml})$. An aliquot of protein extract $(200 \mu \mathrm{g})$ was incubated with the required primary antibody for $4 \mathrm{~h}$ at $4^{\circ} \mathrm{C}$ and incubated overnight at $4^{\circ} \mathrm{C}$ with the appropriate protein $\mathrm{A} / \mathrm{G}$ agarose. Following washing of the immunoprecipitates three times with IP buffer, the pellets were dissolved in gel-loading buffer at a volume ratio of $1: 1$ and boiled for $5 \mathrm{~min}$. Following cooling, the samples were centrifuged at 2,400 $\mathrm{x}$ g for $1 \mathrm{~min}$ and the supernatants were used in subsequent SDS-PAGE analysis, followed by immunoblotting.

Reverse transcription-quantitative polymerase chain reaction (RT-qPCR). Total RNA was isolated from tissues using TRIzol reagent (Invitrogen Life Technologies, Carlsbad, CA, USA) and RT-qPCR was performed as previously described (18). Briefly, cDNA was synthesized from RNA using M-MLV Reverse Transcriptase (Promega Corporation, Madison, WI, USA) at the following temperatures: $60 \mathrm{~min}$ at $42^{\circ} \mathrm{C}$ and $2 \mathrm{~min}$ at $100^{\circ} \mathrm{C}$. cDNA amplification was performed in a PCR master mix containing $1 \mu 1$ reverse transcription reaction product, 1X PCR buffer (Perkin Elmer, Gaithersburg, MD, USA), $0.2 \mathrm{mM}$ dNTP, $0.25 \mathrm{U}$ Taq polymerase (Perkin Elmer), and $50 \mathrm{ng}$ sense and anti-sense primers (Bioneer Corp., Daejeon, Korea). The primers used were as follows: MuRF1 sense, 5'-TTCATCGAGGCCCTGATCCT-3' and anti-sense, 5'-CTTGGCTTCCTTCCCCCTTT-3'; MyHC sense, 5'-TGCCAAGACCGTGAGGAATG-3' and anti-sense, 5'-AATGCATCACAGCTCCCGTG-3'; GAPDH sense, 5'-GGGTGATGCTGGTGCTGAGTATGT-3' and anti-sense, 5'-AAGAATGGgagtTGCTGTTGAAGTC-3'. The PCR reactions were performed in a GeneAmp ${ }^{\circledR}$ PCR system 2400 (Perkin Elmer). The amplified PCR products were electrophoresed on $1 \%$ agarose gels, visualized by ethidium bromide staining, and images were captured using the BioSpectrum ${ }^{\circledR}$ AC Imaging system (UVP, LLC, Upland, CA, USA). The images were imported into TotalLab 1D software (version 12.2; TotalLab Ltd., Newcastle-upon-Tyne, UK) and analyzed.
Statistical analysis. Values are expressed as the mean \pm standard error of the mean and were analyzed using GraphPad Prism (version 5.0; GraphPad Software, Inc., La Jolla, CA, USA). Analysis of variance was used to analyze intergroup differences. Tukey's Multiple Comparison test was used to evaluate differences between values. $\mathrm{P}<0.05$ was considered to indicate a statistically significant difference.

\section{Results}

LE supplementation fails to prevent dexamethasone-induced muscle weight loss. Body weight changes were measured over the five-day experimental period. The dexamethasone-treated groups demonstrated progressive weight loss throughout the experiment compared with the control group (Fig. 1A). To investigate muscle loss following treatment, the weight of soleus and gastrocnemius muscles was measured immediately following biopsy. Dexamethasone significantly decreased the weight of each muscle (Fig. 1B and C) and LE supplementation failed to prevent this muscle weight loss.

LE supplementation improves dexamethasone-induced muscle atrophy by reducing muscle damage. To assess the loss of muscle strength by dexamethasone, forelimb grip strength was measured using a grip strength meter. Dexamethasone treatment gradually reduced grip strength during the experimental period (data not shown) and significantly reduced grip strength on experimental day 5 compared with that in the vehicle-treated control group (Fig. 2A). Of note, supplementation with LE effectively prevented this dexamethasone-induced muscle weakness (Fig. 2A). Since the increment of CK in the serum is considered a surrogate marker of muscle damage, particularly for the diagnosis of myocardial infarction, muscular dystrophy and cerebral diseases (19), the activity of serum CK was determined following 5 days of dexamethasone treatment. Dexamethasone significantly increased the serum CK activity compared with that in the vehicle-treated controls 
A

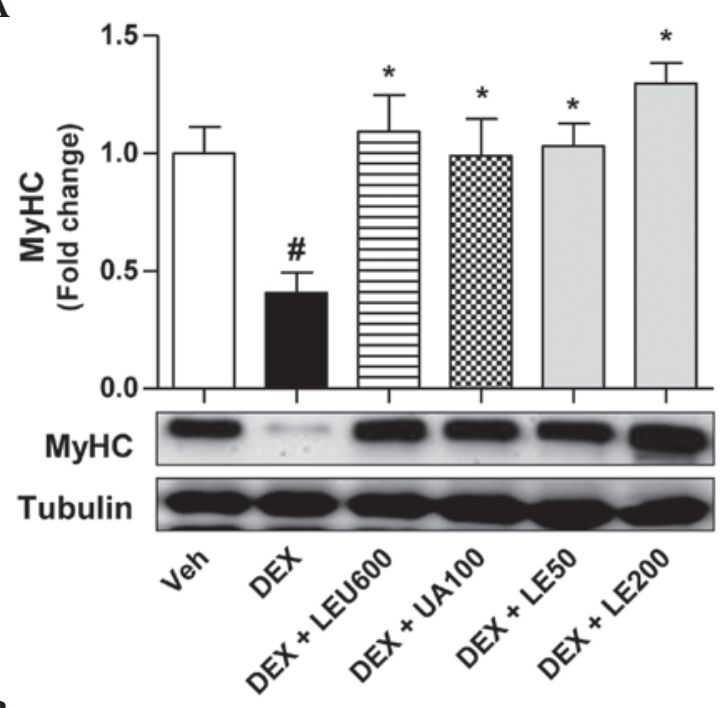

B

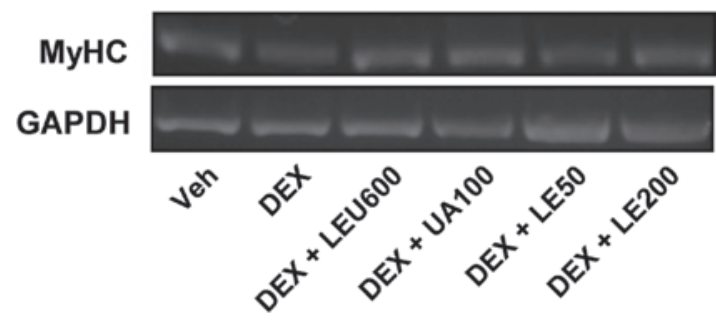

C
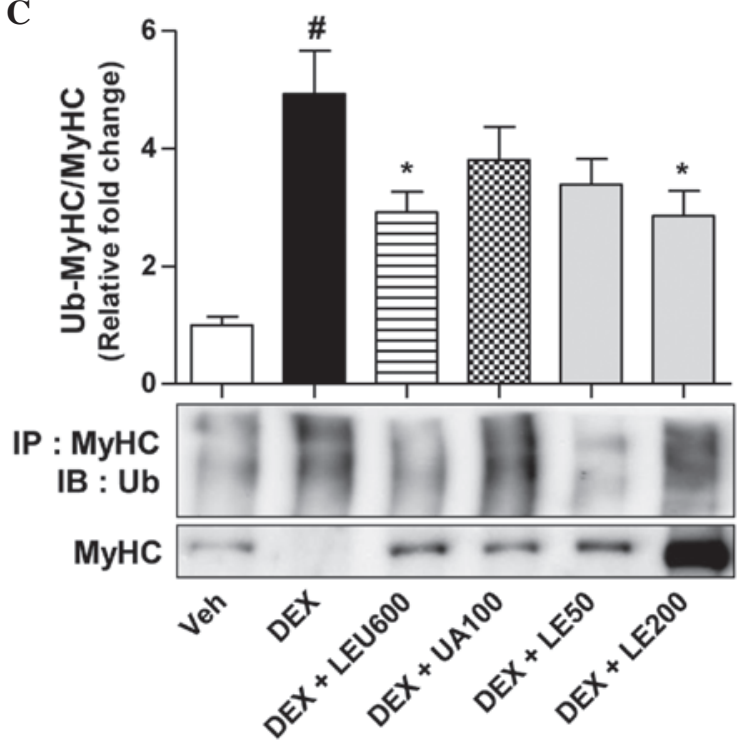

Figure 4. Effects of LE supplementation on DEX-induced MyHC degradation. (A) Representative western blots for MyHC. The western blots were quantified by densitometric analyses and fold changes relative to the Veh group are presented. (B) mRNA levels of MyHC in soleus muscles. (C) Detection of Ub-MyHC by IP. Representative western blot results for Ub-MyHC and $\mathrm{MyHC}$ in each study group. The Ub-MyHC to MyHC ratios are expressed as the fold increase over that of the vehicle-treated group, which was arbitrarily set as 1 . Values are expressed as the mean \pm standard error of the mean. Treatment doses are stated in $\mathrm{mg} / \mathrm{kg}$ body weight. ${ }^{~} \mathrm{P}<0.05$, compared with the DEX group and the Veh group; ${ }^{*} \mathrm{P}<0.05$, compared with the DEX group and the other four groups. DEX, dexamethasone; LEU, L-leucine; UA, ursolic acid; LE, loquat extract; Veh, vehicle-treated; MyHC, myosin heavy chain; IP, immunoprecipitation; IB, immunoblot; Ub, ubiquitin.

(Fig. 2B), while supplementation with LE effectively reduced these dexamethasone-induced increases.
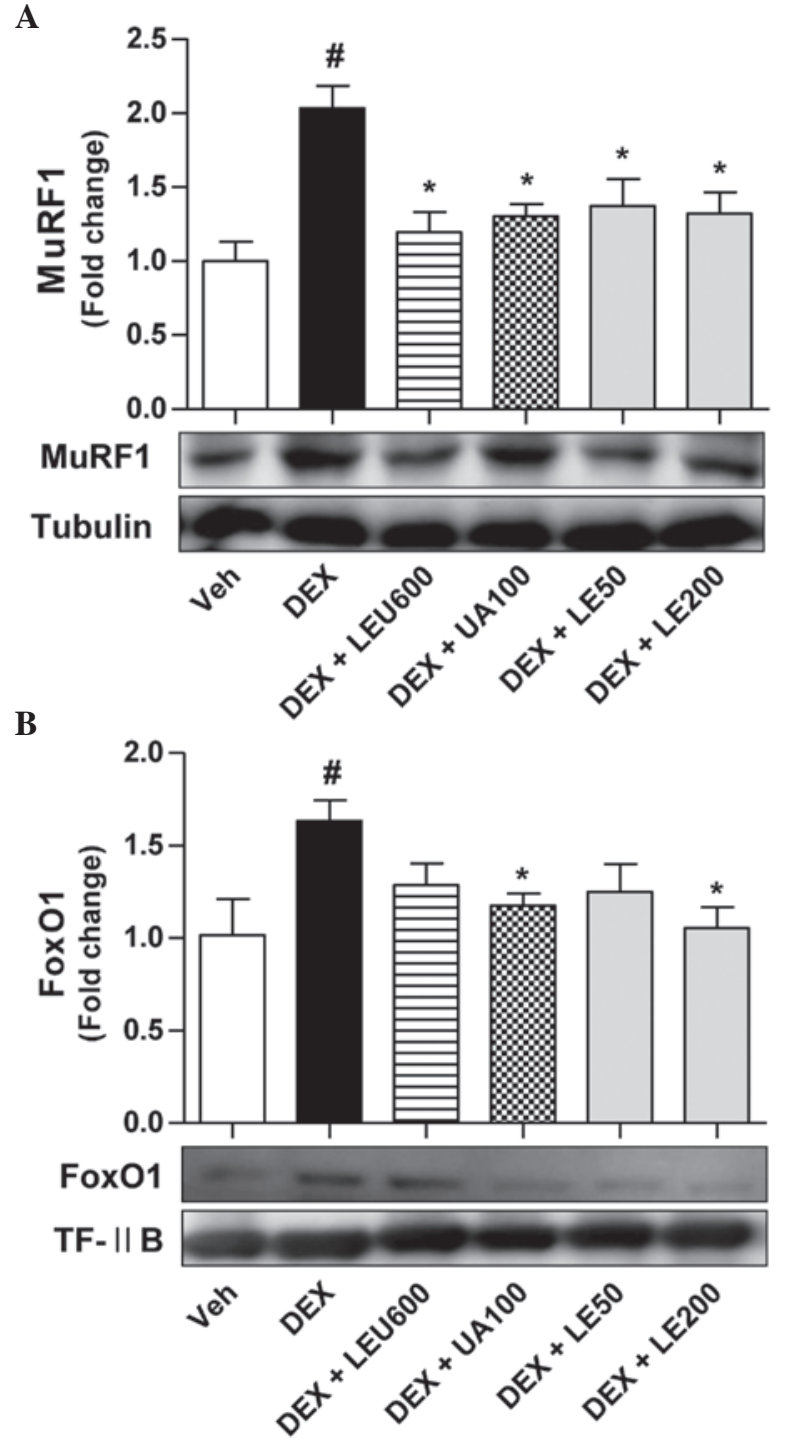

Figure 5. Effects of LE supplementation on the DEX-induced protein expression levels of MuRF1 and in the nuclear translocation of FoxO1. (A) Representative western blots of MuRF1 and (B) nuclear FoxO1 in each study group. The blots were quantified by densitometric analysis and fold changes relative to the Veh group are presented. Values are expressed as the mean \pm standard error of the mean. Treatment doses are stated in $\mathrm{mg} / \mathrm{kg}$ body weight ${ }^{\#} \mathrm{P}<0.05$, compared with the DEX group and the Veh group; ${ }^{*} \mathrm{P}<0.05$, compared with the DEX group and the other four groups. DEX, dexamethasone; LEU, L-leucine; UA, ursolic acid; LE, loquat extract; Veh, vehicle-treated; MuRF1, muscle ring finger 1; Fox, forkhead box protein; TF, transcription factor.

In order to assess the protective effect of LE on dexamethasone-induced muscle damage, histological staining was performed on the medial portions of soleus muscles, using hematoxylin and eosin dyes (H\&E). As shown in Fig. 3A, the muscle fibers in the vehicle-treated group were in close contact in muscle bundles and exhibited relatively uniform fiber diameters (Fig. 3A). However, dexamethasone treatment caused severe damage to the muscle bundles and resulted in an atrophic pattern of muscle fibers (Fig. 3B). The proliferation of connective tissue, particularly surrounding the muscle fibers (endomysium) and fiber bundles (perimysium), as well as angulated and degenerated fibers were observed in the soleus muscle of dexamethasone-treated group (Fig. 3B). However, 
these atrophic damages were ameliorated by supplementation with leucine or ursolic acid (Fig. 3C and D), and to a similar extent by supplementation with LE (Fig. 3E and F). These results suggested that LE supplementation effectively prevented dexamethasone-induced muscle damage and muscle weakness.

LE supplementation reduces the ubiquitin-dependent proteolysis of $\mathrm{MyHC}$. Since it was demonstrated that dexamethasone treatment induced the selective loss of the critical sarcomeric protein MyHC, which is important in muscle contraction (12), the present study assessed the protein expression levels of MyHC in soleus muscles. Dexamethasone markedly reduced the protein expression of MyHC, while LE supplementation effectively inhibited this reduction (Fig. 4A). Therefore, the present study aimed to determine whether the observed reduction in MyHC levels was caused by increased protein degradation or decreased protein synthesis. Neither dexamethasone nor supplementation had any effect on the mRNA expression levels of MyHC (Fig. 4B), which indicated that the decline of MyHC levels was not caused by modulation of MyHC protein synthesis. Since muscle degradation is largely due to the ubiquitination of MyHC, which is catalyzed by E3 ligase (12), MyHC ubiquitination was subsequently detected. Dexamethasone significantly increased the ratio of ubiquitinated MyHC compared with total MyHC (Fig. 4C) and this increase was markedly reduced by LE supplementation (Fig. 4C). These results demonstrated that LE supplementation inhibited dexamethasone-induced muscle loss by reducing the ubiquitination of MyHC.

\section{LE supplementation affects dexamethasone-induced MuRFI} expression by modulating FoxO1. MuRF1 is a specific E3 ligase responsible for the degradation of MyHC (12). It was demonstrated that dexamethasone markedly increased the protein expression levels of MuRF1 (Fig. 5A), which was significantly attenuated by supplementation with LE (Fig. 5A). In addition, the effect of dexamethasone on the nuclear translocation of the transcription factor FoxO1, which induces the expression of MuRF1, was assessed. Dexamethasone was revealed to induce FoxO1 translocation (Fig. 5B) and LE supplementation reduced this effect. These results suggested that LE supplementation reduces the expression of MuRF1 by inhibiting the nuclear translocation of FoxO1.

\section{Discussion}

Skeletal muscle atrophy has limited treatment options and positive clinical outcomes, compromises the quality of life and increases morbidity and mortality $(20,21)$. It has been established that the imbalance between the rates of protein synthesis and breakdown contributes to muscle atrophy. In previous studies, ubiquitin-dependent proteolysis was revealed to be pivotal in muscle protein breakdown $(9,22)$, and therefore, the modulation of protein breakdown during muscle atrophy represents an interesting therapeutic strategy. Several agents have been identified and developed for this purpose. In particular, ursolic acid, which is a lipophilic pentacyclic triterpene, has a potent effect on muscle atrophy (8), and ursolic acid is present in several plants, including the leaves of

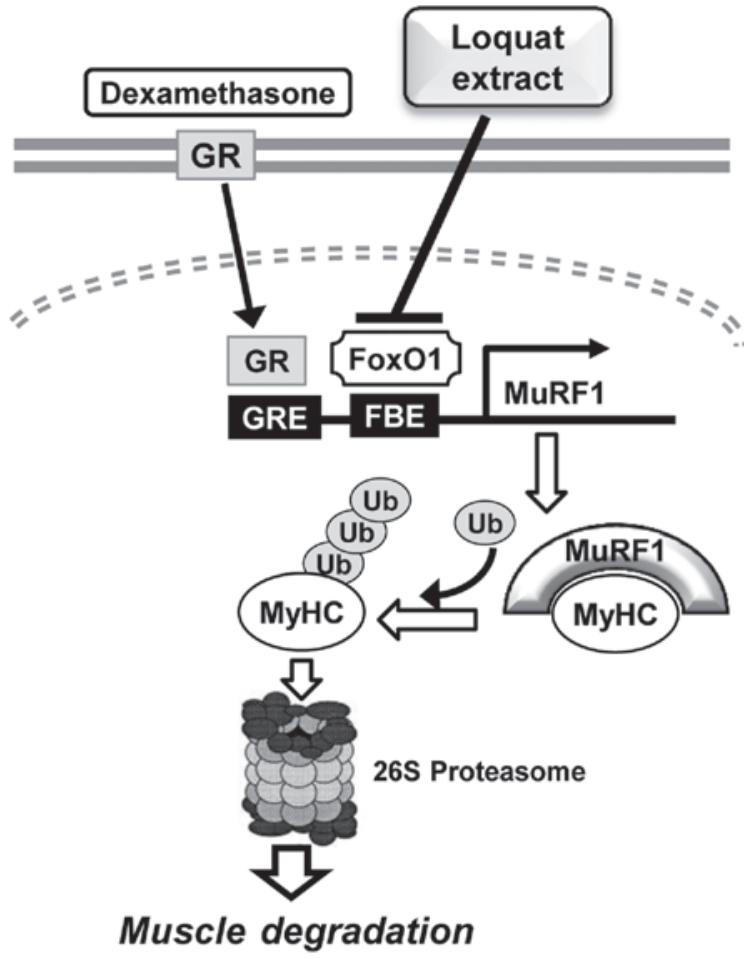

Figure 6. Suggested mechanism for the effect of LE on DEX-induced muscle atrophy. LE inhibited the translocation of FoxO1. Consequently, the expression levels of MuRF1, which is responsible for muscle atrophy, was downregulated. Downregulation of MuRF1 is associated with ubiquitination and degradation of $\mathrm{MyHC}$, which is an important component of muscle fiber. FBE, FoxO binding element; FoxO, forkhead box O; GR, glucocorticoid receptor; GRE, glucocorticoid response element; MuRF1, muscle ring finger 1; MyHC, myosin heavy chain; Ub, ubiquitin.

loquat (5). Therefore, the present study investigated the effect of LE on muscle atrophy.

In the present study, the preventive effects of LE on dexamethasone-induced muscle atrophy were assessed in Sprague Dawley rats. Previous studies used glucocorticoids to induce muscle atrophy in rodents, and it was demonstrated to cause catabolic states and physiological muscle changes $(12,23)$. For example, the glucocorticoid dexamethasone significantly induced muscle wasting and altered the balance between protein synthesis and degradation $(12,15,24)$. The findings of the present study regarding the effects of dexamethasone concur with those of these previous studies. In the present study, LE had no effect on the decreases in body weight induced by dexamethasone; however, LE supplementation significantly inhibited dexamethasone-induced reductions in muscle strength. In a previous study, other markers of muscle atrophy were altered without body or muscle weight gain in dexamethasone-treated rats (25). These findings suggested that muscle mass is not affected by LE administration, however, that LE has a beneficial effect on muscle strength.

To confirm the effects of LE, the muscle damage induced by dexamethasone was investigated using two methods. It has been reported that the appearance of CK in blood serum is a classical marker of muscle breakdown (26). In the present study, CK levels were increased by dexamethasone and LE supplementation abolished these increases. Although CK is not a specific marker of muscle breakdown, the results 
indicated the possibility that the muscle breakdown induced by dexamethasone was attenuated by LE. To confirm this assumption, histological analysis was performed by $\mathrm{HE}$ staining. It was previously reported that non-damaged muscle fibers are tight and in close contact with each other in muscle bundles, however, that damaged muscle fibers are not (27). In the present study, LE supplementation was observed to reduce muscle damage caused by dexamethasone administration, which suggested that the muscle damage induced by dexamethasone was diminished by LE.

In catabolic states, insulin signaling is not sufficient to inhibit FoxO factors $(21,28,29)$. Therefore, transactivation of FoxO results in the expression of MuRF1, which leads to the ubiquitination and degradation of MyHC (12,14,15). Since the administration of dexamethasone is known to influence this signaling and to induce muscle protein degradation and synthesis imbalance $(14,15)$, the present study investigated whether these signals were affected by LE. Western blot analysis demonstrated that the protein levels of MyHC were downregulated by dexamethasone and that LE inhibited this downregulation. However, the mRNA expression levels of MyHC were identical in all of the groups, indicating that the reduction in the protein expression of MyHC was caused by protein degradation, rather than transcription. Since MyHC is degraded by a proteasome-dependent pathway (12), the ubiquitination of MyHC was assessed. This revealed that the dexamethasone-induced ubiquitination of MyHC was markedly reduced by LE supplementation. MuRF1 is known to catalyze the ubiquitination and degradation of $\mathrm{MyHC}$ (12), and therefore, the present study next assessed the protein expression levels of MuRF1 in the soleus muscle. As expected, the protein expression levels of MuRF1 were increased by dexamethasone, but suppressed by LE. Whether LE modulated the transcriptional activity of FoxO1 was determined by western blot analysis using the nuclear fraction. The levels of FoxO1 in the nucleus were increased by dexamethasone and these increases were inhibited by LE, which was consistent with the observations regarding the expression of MuRF1. These findings suggested that LE inhibited the ubiquitin-dependent proteolysis of MyHC by inhibiting the translocation of FoxO1, and therefore, modulating the expression of MuRF1. The scheme in Fig. 6 illustrates the mechanism of action of LE according to the findings of the present study.

In conclusion, LE was demonstrated to alleviate dexamethasone-induced muscle atrophy by inhibiting the translocation of FoxO1, downregulating MuRF1 and therefore, reducing the degradation of MyHC. The present study has provided evidence for the efficacy of LE, which has long been used in folk medicine in the treatment of muscle atrophy, and elucidated its underlying mechanism of action. Based on these findings, LE is recommended to be used as a chemopreventive agent against muscle aging and as a therapeutic agent against skeletal muscle atrophy in the elderly.

\section{Acknowledgements}

This study was supported by the National Research Foundation of Korea grant funded by the Korea government (no. 2009-0083538) and by the R\&D program of MOTIE/KEIT (no. 10040391; Development of Functional Food Materials and Device for the Prevention of Aging-associated Muscle Function Decrease). In addition, the authors would like to thank the Aging Tissue Bank for providing research information.

\section{References}

1. Banno N, Akihisa T, Tokuda $\mathrm{H}$, et al: Anti-inflammatory and antitumor-promoting effects of the triterpene acids from the leaves of Eriobotrya japonica. Biol Pharm Bull 28: 1995-1999, 2005.

2. Uto T, Suangkaew N, Morinaga O, Kariyazono H, Oiso S and Shoyama Y: Eriobotryae folium extract suppresses LPS-induced iNOS and COX-2 expression by inhibition of NF-kappaB and MAPK activation in murine macrophages. Am J Chin Med 38: 985-994, 2010

3. De Tommasi N, De Simone F, Pizza C, et al: Constituents of Eriobotrya japonica. A study of their antiviral properties. J Nat Prod 55: 1067-1073, 1992.

4. Shimizu M, Uemitsu N, Shirota M, Matsumoto K and Tezuka Y: A new triterpene ester from Eriobotrya japonica. Chemical \& Pharmaceutical Bulletin 44: 2181-2182, 1996.

5. Chen Q, Zhang Y, Zhang W and Chen Z: Identification and quantification of oleanolic acid and ursolic acid in Chinese herbs by liquid chromatography-ion trap mass spectrometry. Biomed Chromatogr 25: 1381-1388, 2011.

6. Hsu YL, Kuo PL and Lin CC: Proliferative inhibition, cell-cycle dysregulation, and induction of apoptosis by ursolic acid in human non-small cell lung cancer A549 cells. Life Sci 75: 2303-2316, 2004

7. Wang X, Zhang F, Yang L, et al: Ursolic acid inhibits proliferation and induces apoptosis of cancer cells in vitro and in vivo. J Biomed Biotechnol 2011: 419343, 2011.

8. Kunkel SD, Suneja M, Ebert SM, et al: mRNA expression signatures of human skeletal muscle atrophy identify a natural compound that increases muscle mass. Cell Metab 13: 627-638, 2011.

9. de Palma L, Marinelli M, Pavan M and Orazi A: Ubiquitin ligases MuRF1 and MAFbx in human skeletal muscle atrophy. Joint Bone Spine 75: 53-57, 2008.

10. Foletta VC, White LJ, Larsen AE, Leger B and Russell AP: The role and regulation of MAFbx/atrogin-1 and MuRF1 in skeletal muscle atrophy. Pflugers Arch 461: 325-335, 2011.

11. Witt SH, Granzier H, Witt CC and Labeit S: MURF-1 and MURF-2 target a specific subset of myofibrillar proteins redundantly: towards understanding MURF-dependent muscle ubiquitination. J Mol Biol 350: 713-722, 2005.

12. Clarke BA, Drujan D, Willis MS, et al: The E3 Ligase MuRF1 degrades myosin heavy chain protein in dexamethasone-treated skeletal muscle. Cell Metab 6: 376-385, 2007.

13. Tintignac LA, Lagirand J, Batonnet S, Sirri V, Leibovitch MP and Leibovitch SA: Degradation of MyoD mediated by the SCF (MAFbx) ubiquitin ligase. J Biol Chem 280: 2847-2856, 2005.

14. Sandri M, Sandri C, Gilbert A, et al: Foxo transcription factors induce the atrophy-related ubiquitin ligase atrogin-1 and cause skeletal muscle atrophy. Cell 117: 399-412, 2004.

15. Waddell DS, Baehr LM, van den Brandt J, et al: The glucocorticoid receptor and FOXO1 synergistically activate the skeletal muscle atrophy-associated MuRF1 gene. Am J Physiol Endocrinol Metab 295: E785-E797, 2008.

16. Kamei Y, Miura S, Suzuki M, et al: Skeletal muscle FOXO1 (FKHR) transgenic mice have less skeletal muscle mass, down-regulated Type I (slow twitch/red muscle) fiber genes, and impaired glycemic control. J Biol Chem 279: 41114-41123, 2004.

17. Jung HA, Park JC, Chung HY, Kim J and Choi JS: Antioxidant flavonoids and chlorogenic acid from the leaves of Eriobotrya japonica. Arch Pharm Res 22: 213-218, 1999.

18. Noh KK, Chung KW, Choi YJ, et al: beta-Hydroxy beta-methylbutyrate improves dexamethasone-induced muscle atrophy by modulating the muscle degradation pathway in SD Rat. PLoS One 9: e102947, 2014.

19. Magal M, Dumke CL,Urbiztondo ZG, et al: Relationship between serum creatine kinase activity following exercise-induced muscle damage and muscle fibre composition. J Sports Sci 28: 257-266, 2010.

20. Holecek M: Muscle wasting in animal models of severe illness. Int J Exp Pathol 93: 157-171, 2012. 
21. Morley JE: Sarcopenia in the elderly. Fam Pract 29 (Suppl 1): i44-i48, 2012.

22. Stitt TN, Drujan D, Clarke BA, et al: The IGF-1/PI3K/Akt pathway prevents expression of muscle atrophy-induced ubiquitin ligases by inhibiting FOXO transcription factors. Mol Cell 14: 395-403, 2004.

23. Romanick M, Thompson LV and Brown-Borg HM: Murine models of atrophy, cachexia, and sarcopenia in skeletal muscle. Biochim Biophys Acta 1832: 1410-1420, 2013.

24. Schakman O, Gilson H and Thissen JP: Mechanisms of glucocorticoid-induced myopathy. J Endocrinol 197: 1-10, 2008.

25. Yamamoto D, Maki T, Herningtyas EH, et al: Branched-chain amino acids protect against dexamethasone-induced soleus muscle atrophy in rats. Muscle Nerve 41: 819-827, 2010.
26. Gehrig SM, van der Poel C, Sayer TA, et al: Hsp72 preserves muscle function and slows progression of severe muscular dystrophy. Nature 484: 394-398, 2012.

27. Toumi H, Hegge J, Subbotin V, et al: Rapid intravascular injection into limb skeletal muscle: a damage assessment study. Mol Ther 13: 229-236, 2006.

28. Brunet A, Bonni A, Zigmond MJ, et al: Akt promotes cell survival by phosphorylating and inhibiting a Forkhead transcription factor. Cell 96: 857-868, 1999.

29. Kops GJ, de Ruiter ND, De Vries-Smits AM, Powell DR, Bos JL and Burgering BM: Direct control of the Forkhead transcription factor AFX by protein kinase B. Nature 398: 630-634, 1999. 